\title{
Factors Affecting Early Postoperative Visual Acuity in Cataract Patients with High Myopia
}

\author{
Rongrong Zhang ${ }^{1 \#}$, Fu Li, MD ${ }^{2 \#}$, Yan Yu ${ }^{1}$, Tiantian Wang ${ }^{1}$, Changfan $W^{1^{*}}$ \\ ${ }^{1}$ Department of Ophthalmology, Yijishan Hospital of Wannan Medical College, China \\ ${ }^{2}$ Department of Ophthalmology, Aier Eye Hospital (HEZE) of Heze City, China

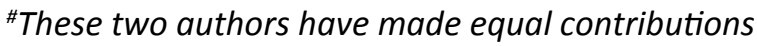

*Corresponding author: Changfan Wu, Department of Ophthalmology, Yijishan Hospital of Wannan Medical College, Wuhu 241001, China, Tel: +86-139-0963-2351, Fax: +86-553-5738279

\begin{abstract}
Purpose: To analyze the short-term results of cataract surgery in patients with high myopia and determine the risk factors responsible for early postoperative visual outcome.

Methods: A total of 93 patients (115 eyes) with high myopia who underwent phacoemulsification and intraocular lens (IOL) implantation were enrolled in this retrospective study. Complete ophthalmologic examinations were performed preoperatively and postoperatively. According to the best-corrected visual acuity (BCVA) at one week after surgery, all patients were divided into Group $A$ (postoperative BCVA $\geq 20 / 40$ ) and Group B (postoperative BCVA $<20 / 40$ ) for comparison and to identify the related factors of early postoperative vision. The difference between the two groups was analyzed with independent t-text. Logistic regression was utilized to analyze age, axial length $(A L)$, maculopathy and other related factors for early postoperative BCVA.
\end{abstract}

Results: Among the 115 eyes, group A were 66 eyes and group B were 49 eyes. In multivariate regression analysis, maculopathy (OR: $5.586,95 \% \mathrm{Cl}: 1.704-18.311$ ), posterior staphyloma (OR: 3.698, 95\% Cl: 1.002-13.652) and preoperative visual acuity (VA) (OR: $4.835,95 \% \mathrm{Cl}: 1.962-$ $11.915)$ were associated with early postoperative BCVA in cataract with high myopia (all $p<0.05$ ), while not associated with age, $A L$ and other factors (all $p>0.05$ ).

Conclusion: Most of the eyes with high myopia achieved satisfactory improvement in postoperative BCVA. In the short-term study, the lower the preoperative VA, pre-existing maculopathy or posterior staphyloma are risk factors for early postoperative BCVA recovery in cataract patients with high myopia.

\section{Keywords}

Cataract, High myopia, Cataract surgery, Early postoperative BCVA, Factors

\section{Introduction}

Myopia and its complications is a major cause of visual impairment in many countries [1]. Epidemiological studies showed that the prevalence rate of high myopia from 2000 to 2050 was $2.7 \%-9.8 \%$, showing an increasing trend year by year [2]. And the prevalence of high myopia in the Asian population was higher than that of non-Asian population, especially in China [3-5].

Highly myopic eyes not only may more commonly have coexisting ocular conditions [6], such as retinal tears, lattice degeneration and liquefied vitreous but also a risk factor for cataract formation. With the increase of the high myopia population, the prevalence of cataract with high myopia gradually increased and the age at which cataracts developed was earlier. Several studies have shown that nuclear cataracts were more common than cortical cataracts in patients with high myopia. The cross-section data from the Blue Mountains Eye Study demonstrated a strong association between high myopia and nuclear cataracts and posterior subcapsular cataracts (PSC) [7]. Praveen, et al. [8] in a prospective case-control study of 800 eyes showed an association between nuclear cataracts and axial high myopia. Cataract surgery was among the most fre-

Citation: Zhang R, Li F, Yu Y, Wang T, Wu C (2020) Factors Affecting Early Postoperative Visual Acuity in Cataract Patients with High Myopia. Int J Ophthalmol Clin Res 7:117. doi.org/10.23937/2378346X/1410117

Accepted: August 20, 2020: Published: August 22, 2020

Copyright: (c) 2020 Zhang R, et al. This is an open-access article distributed under the terms of the Creative Commons Attribution License, which permits unrestricted use, distribution, and reproduction in any medium, provided the original author and source are credited. 
quently performed surgical processes $[9,10]$, but which in highly myopic eyes was more complex than in healthy eyes and had a considerable potential for an unfavorable postoperative outcome.

Previous studies have assessed the risk factors, which could influence the outcome of cataract surgery in highly myopic eyes during long-term follow-up. Xu, et al. [11] and Zuberbuhler, et al. [12] found that postoperative visual acuity was significantly related to chorioretinal degeneration, age, and AL. Pre-existing maculopathy was also found to be an independent risk factor for failure to achieve good postoperative vision [13]. However, few studies have investigated the association between risk factors and postoperative BCVA during a short-term period, which would affect patients' satisfaction after cataract surgery. So, this study aims to investigate the short-term visual outcomes after cataract surgery in highly myopic patients and to further estimate the associated risk factors of early postoperative BCVA.

\section{Methods}

This study recorded 93 patients (115 eyes) with high myopia who underwent phacoemulsification and IOL implantation retrospectively reviewed October 2015 and September 2017. Only patients who meet the diagnostic criteria for cataracts and $A L \geq 26.0 \mathrm{~mm}$ were included in this study. For the present analysis, eyes with a history of surgery for retinal detachment or a history of trauma, as well as glaucoma, retinal disease, keratitis and other eye diseases that seriously affect postoperative vision should be excluded. According to the BCVA at one week after operation, all patients were divided into Group A (defined as postoperative BCVA $\geq 20 / 40$ ) and Group B (postoperative BCVA < 20/40) for comparison and to identify the related factors of early postoperative vision. This study followed the tenets of the Declaration of Helsinki, and approval was obtained from the Ethics Committee of our hospital. At the time of the surgery, all patients were fully informed about the details and possible risks of the surgical procedure. Informed consent was signed by all patients before surgery.

All patients underwent the standard comprehensive anterior and posterior segment examination procedure preoperatively and postoperatively which were performed by trained and professional ophthalmologists. Visual acuity was tested with a Snellen E-chart at $6 \mathrm{~m}$ by ophthalmic assistants. Intraocular pressure was measured with a Non-contact tonometer at every visit. An auto refractometer (ARK-710A Autorefractor, NIDEK, Japan) was used for keratometry measurement and objective refraction. An optical biometer (IOLMaster, Zeiss) was performed for AL measurement. But, the AL was determined with fixation using A-scan ultrasound biometry (CineScan, Quamtel France), when it could not be obtained or if a staphyloma was detected. B-scan ultrasonography was used for anterior chamber depth (ACD) and posterior staphyloma measurements. An optical coherence tomography (OCT) device (Spectralis, Heidelberg, Germany) was applied for fundus imaging. Blood pressure (BP) was measured by a blood pressure meter. Height and weight were measured to calculate $\mathrm{BMI}$ (BMI = The square of weight divided by height).

All the surgeries were carried out by two surgeons. A $2.6 \mathrm{~mm}$ clear corneal tunnel incision was made after local anesthesia. After injection of $1 \%$ sodium hyaluronate into anterior chamber, continuous curvilinear capsulorhexis, hydrodissection and hydrodelineation, nucleus chopping, and phacoemulsification were performed, followed by aspiration of the residual cortex, and insertion of a foldable IOL into the bag or into the sulcus. The corneal incisions were hydrated after adjusting the position of the IOL and removing the viscoelastics. Routine follow-up schedule for uneventful surgery included examination on postoperative day 1 and week 1. Postoperative examinations included slit lamp, visual acuity assessment, manifest refraction and fundus were performed by ophthalmologists.

The quantitative data was described by mean \pm standard deviation, and $t$ test was used for the comparison among groups. The counting data was expressed by frequency, and the intergroup comparison was tested by $\chi^{2}$ tests. Multiple logistic regressions performed for multivariate analysis to reveal the impact of individually associated risk factors according to early postoperative BCVA. $P<0.05$ was statistically significant. The statistical analysis was performed using SPSS for windows version of the 22.0 (SPSS Inc., Chicago, IL).

\section{Results}

The analysis included 115 eyes from 93 patients with a mean age of $62.73 \pm 11.03$ years. Group A were 66 eyes (57.4\%) and Group B were 49 eyes (42.6\%). And early postoperative complications were observed in 3 eyes $(2.61 \%)$. Corneal edema and hyphema were respectively occurred in 2 eyes (1.74\%) and 1 eyes ( $0.87 \%)$.

Table 1 showed the demographic data analysis of cataract patients with high myopia. There were significant differences in age, AL and ACD ( $p=0.023, p=0.035$, $p=0.001$, respectively), but no significantly different in sex, BMI, BP and keratometry $(p>0.05)$ between group $A$ and group $B$. The factors of maculopathy, posterior staphyloma and preoperative VA between group $A$ and group $B$ were also significant differences $(p=0.001, p=$ $0.004, p=0.001$, respectively).

Table 2 showed the results of multivariate regression analysis of risk factors for early postoperative BCVA in cataract patients with high myopia. The maculopathy (OR: $5.586,95 \% \mathrm{Cl}: 1.704-18.311$ ), posterior staphyloma (OR: $3.698,95 \% \mathrm{Cl}: 1.002-13.652$ ) and preoperative VA (OR: $4.835,95 \% \mathrm{Cl}: 1.962-11.915)$ were related to early postoperative BCVA (all $p<0.05$ ) and were the independent risk factors of postoperative visual acuity recovery. Patients in group A had lower preoperative VA 
Table 1: Demographic data analysis of cataract patients with high myopia.

\begin{tabular}{|c|c|c|c|c|c|}
\hline Factors & Group A & Group B & $\mathbf{t}$ & $x^{2}$ & P-value \\
\hline Age(years) & $65.37 \pm 9.76$ & $60.77 \pm 11.57$ & 2.306 & & 0.023 \\
\hline \multicolumn{6}{|l|}{ Sex } \\
\hline Male & 15 & 25 & & & \\
\hline Female & 34 & 41 & & 0.655 & 0.418 \\
\hline BMI $\left(k g / m^{2}\right)$ & $23.75 \pm 3.90$ & $22.95 \pm 2.80$ & 0.655 & & 0.418 \\
\hline SBP $(\mathrm{mmHg})$ & $126.49 \pm 18.20$ & $124.77 \pm 14.27$ & 0.567 & & 0.572 \\
\hline DBP (mmHg) & $78.71 \pm 9.26$ & $76.82 \pm 9.54$ & 1.067 & & 0.288 \\
\hline K (D) & $44.80 \pm 2.99$ & $44.39 \pm 2.50$ & 0.811 & & 0.419 \\
\hline $\mathrm{AL}(\mathrm{mm})$ & $29.88 \pm 1.83$ & $29.14 \pm 1.83$ & 2.128 & & 0.035 \\
\hline ACD (mm) & $3.17 \pm 0.51$ & $3.46 \pm 0.44$ & -3.264 & & 0.001 \\
\hline \multicolumn{6}{|l|}{ Pre-VA (D) } \\
\hline$<0.1$ & 47 & 45 & & & \\
\hline $0.1-0.3$ & 2 & 19 & & & \\
\hline$>0.3$ & 0 & 2 & & 44.365 & 0.000 \\
\hline \multicolumn{6}{|l|}{ Maculopathy } \\
\hline Yes & 41 & 19 & & & \\
\hline No & 8 & 47 & & 33.950 & 0.000 \\
\hline \multicolumn{6}{|l|}{ PS } \\
\hline Yes & 40 & 37 & & & \\
\hline No & 9 & 29 & & 8.312 & 0.004 \\
\hline
\end{tabular}

Abbreviations: BMI: Body Mass Index; SBP: Systolic Blood Pressure; DBP: Diastolic Blood Pressure; K: keratometry; D: Diopter; AL: Axial Length; ACD: Anterior Chamber Depth; Pre-VA: Preoperative Visual Acuity; PS: Posterior Staphyloma.

Table 2: Multivariate logistic regression analysis of early postoperative BCVA.

\begin{tabular}{|l|l|l|l|l|}
\hline & Wald & OR & 95\%Cl & P-value \\
\hline Age (years) & 1.359 & 0.966 & $0.913-1.024$ & 0.244 \\
\hline AL (mm) & 0.017 & 0.976 & $0.686-1.390$ & 0.895 \\
\hline ACD (mm) & 3.124 & 2.837 & $0.893-9.019$ & 0.077 \\
\hline Pre-VA (D) & 11.728 & 4.835 & $1.962-11.915$ & 0.001 \\
\hline Maculopathy & 8.067 & 5.586 & $1.704-18.311$ & 0.005 \\
\hline PS & 3.853 & 3.698 & $1.002-13.652$ & 0.041 \\
\hline
\end{tabular}

Abbreviations: AL: Axial Length; ACD: Anterior Chamber Depth; Pre-VA: Preoperative Visual Acuity; D: diopter; PS: Posterior Staphyloma.

and were more likely to have maculopathy and posterior staphyloma than those in group $B$.

\section{Discussion}

In the present study, we found that all patients achieved an improvement in visual impairment after cataract surgery. Compared with patients in group $A$, the patients in group $B$ had older age, longer $A L$, deeper anterior chamber, poor preoperative vision and a greater chance of maculopathy and posterior staphyloma. In addition, pre-existing maculopathy or posterior staphyloma and preoperative VA were considered the principal risk factors associated with failure to achieve satisfactory early postoperative BCVA.

Previous studies have reported that the presence of maculopathy has been highlighted as an important risk factor for poor visual outcome after cataract sur- gery $[12,14,15]$. Tsai, et al. [15] in a study of 52 highly myopic eyes performed that pre-existing maculopathy was an independent risk factor for poor postoperative vision during a 6-month follow-up (OR: 6.84, 95\% Cl: 1.12-41.8). This was consistent with our results that the maculopathy was an independent risk factors for early postoperative BCVA in 1 week follow-up (OR: 5.586, $95 \% \mathrm{Cl}: 1.704-18.311)$. As one of the main causes of myopic maculopathy, posterior staphyloma affects the postoperative BCVA by changing the fundus. Wang, et al. [16] in a long-term follow-up study found that the postoperative VA of high myopia patients with posterior staphyloma was significantly lower than that without posterior staphyloma. This outcome was consistent with our study, which was researched in a short-term follow-up. However, in the long-time follow-up study results, the postoperative VA may be caused by the de- 
velopment of fundus lesions or the occurrence of postoperative complication posterior capsule opacification (PCO), while the short-time follow-up study can largely avoid the above effects, and then can get more clear satisfaction of patients. Therefore, early preoperative detection and treatment of possible pathological ocular complications were crucial in the management of cataracts with high myopia. Optical coherence tomography (OCT) was a modern, non-invasive imaging method that had vastly enhanced our ability to study and detect changes at different levels of the posterior pole [17]. Routine preoperative and postoperative assessment of OCT and other fundus examinations should be carried out to keep abreast of the progress of the fundus.

In the present study, we also performed that the preoperative VA grade was an independent risk factor for early postoperative BCVA. This was consistent with a similar study by Zhao and colleagues [18] which reported that preoperative VA was an important index to predict the postoperative vision of cataract patients with high myopia. Theoretically, preoperative VA was a common indicator of the severity of the cataract and the degree of fundus lesions. Patients with good preoperative VA had light cataract and the degree of fundus lesions was also light, so the postoperative visual recovery was good [19]. Therefore, the highly myopic patients complicated with cataracts should be regularly examined by diagnostic optometry.

In general, increasing age and AL were relevant risk factors related to the appearance of pathologic alterations in highly myopic patients $[11,20,21]$. $\mathrm{Ku}$, et al. [20] in a study of 125 highly myopic eyes performed that younger age and shorter AL were appreciated for the better visual outcomes $(p=0.004$ and $p=0.021$, respectively). However, we found no association between age, $A L$ and postoperative BCVA in multivariate regression analysis. And Akar, et al. [13] in a study of 209 highly myopic eyes also reported that patient's age and AL at first seemed to have a negative impact, but after independent risk analysis, they did not have any statistically significant influence on postoperative BCVA ( $p=0.173$ and $p=0.111$, respectively). This conclusion was consistent with us. We concluded that the increase of age and $A L$ mainly causes the fundus change and then leads to the decrease of visual acuity, which may be due to the result of the interaction of various factors.

Some studies reported a positive association between BMI and blood pressure in healthy people $[22,23]$. Otherwise, there was also a strong relationship between BMI and central corneal thickness [24]. Therefore, BMI and blood pressure were included in this study to observe whether they could change the biological parameters of the cornea and then affect the postoperative BCVA. However, we did not observe that $B M I, B P, A C D$, keratometry were associated with early postoperative BCVA in cataract with high myopia.
The limitations of this study were the retrospective study design and the limited sample size. Nonetheless, we provided the short-term visual outcomes and risk factor analysis of cataract surgery in highly myopic patients. A prospective case-control study will be suggested in the future.

\section{Conclusion}

In conclusion, this study investigated the risk factors associated with postoperative BCVA recovery in cataract with high myopia during a short-term phase and gives an overview of the results and associations. Under the premise of cataract diagnosis, patients with poor vision need to have a thorough and comprehensive preliminary fundus examination before surgery. Through this careful examination, the relative risk factors can be found, which can provide more accurate evidence for predicting the recovery of early postoperative vision. So that patients can get the ideal expectations.

\section{Acknowledgments}

The authors would like to thank the contributions of the faculty and staff at the ophthalmology of Yijishan Hospital who made the work described here possible.

\section{References}

1. Sperduto RD, Seigel D, Roberts J, Rowland M (1983) Prevalence of myopia in the United States. Arch Ophthalmol 101: 405-407.

2. Holden BA, Fricke TR, Wilson DA, Jong M, Naidoo KS, et al. (2016) Global Prevalence of Myopia and High Myopia and Temporal Trends from 2000 through 2050. Ophthalmology 123: 1036-1042.

3. Wong YL, Saw SM (2016) Epidemiology of Pathologic Myopia in Asia and Worldwide. Asia Pac J Ophthalmol (Phila) 5: 394-402.

4. Kempen JH, Mitchell P, Lee KE, Tielsch JM, Broman AT, et al. (2004) The prevalence of refractive errors among adults in the United States, Western Europe, and Australia. Arch Ophthalmol 122: 495-505.

5. Gao LQ, Liu W, Liang YB, Zhang F, Wang JJ, et al. (2011) Prevalence and characteristics of myopic retinopathy in a rural Chinese adult population: the Handan Eye Study. Arch Ophthalmol 129: 1199-1204.

6. Hyman L (2007) Myopic and hyperopic refractive error in adults: an overview. Ophthalmic Epidemiol 14: 192-197.

7. Lim R, Mitchell P, Cumming RG (1999) Refractive associations with cataract: the Blue Mountains Eye Study. Investi Ophthalmol Vis Sci 40: 3021-3026.

8. Praveen MR, Shah GD, Vasavada AR, Mehta PG, Gilbert C, et al. (2010) A study to explore the risk factors for the early onset of cataract in India. Eye 24: 686-694.

9. Batterbury M, Khaw PT, Hands R, Elkington AR (1991) The cataract explosion: the changing pattern of diagnoses of patients attending an ophthalmic outpatient department. Eye 5: 369-372.

10. McCarty CA, Keeffe JE, Taylor HR (1999) The need for cataract surgery: projections based on lens opacity, visual acuity, and personal concern. Br J Ophthalmol 83: 62-65. 
11. Xu L, Li Y, Wang S, Wang Y, Wang Y, et al. (2007) Characteristics of highly myopic eyes: the Beijing Eye Study. Ophthalmology 114: 121-126.

12. Zuberbuhler B, Seyedian M, Tuft S (2009) Phacoemulsification in eyes with extreme axial myopia. J Cataract Refract Surg 35: 335-340.

13. Akar S, Gok K, Bayraktar S, Kaya V, Kucuksumer Y, et al. (2010) Phacoemulsification in high myopia. Saudi Med J 31: 1141-1145.

14. Pham TQ, Cugati S, Rochtchina E, Mitchell $P$, Maloof $A$, et al. (2007) Age-related maculopathy and cataract surgery outcomes: visual acuity and health-related quality of life. Eye 21: 324-330.

15. Tsai CY, Chang TJ, Kuo LL, Chou P, Woung LC (2008) Visual outcomes and associated risk factors of cataract surgeries in highly myopic Taiwanese. Ophthalmologica 222: 130-135.

16. Wang $X X$, Zhao GQ, Wang $Q$, Che $C Y$, Zhang $Q Q$, et al. (2012) Visual quality analysis of high myopia with posterior staphyloma after cataract surgery. Int Eye Sci 12: 10111015.

17. Giovinco G, Savastano MC, Ventre S, Tamburrino A (2015) Automated detection of the retinal from OCT spectral domain images of healthy eyes. J Mod Opt 62: 837-850.

18. Zhao YE, Wang QM, Qu J, Lü F, Chen SH, et al. (2003)
The visual outcome and related factors in cataract patients with extreme myopia. [Zhonghua yan ke za zhi] Chinese J Ophthalmol 39: 537-540.

19. Wang JD, Zhang JS, Li J, Wang NL, Xiong Y, et al. (2014) Visual outcomes and related factors in cataract patients with high myopia after phacoemulsification and intraocular lenes implantation. Chinese Journal of Optometry Ophthalmology \& Visual Science 16: 679-681.

20. Ku WC, Chuang LH, Lai CC (2002) Cataract extraction in high myopic eyes. Chang Gung Med J 25: 315-320.

21. Lyle WA, Jin GJC (1996) Phacoemulsifcation with intraocular lens implantation in high myopia. J Cataract Refract Surg 22: 238-242.

22. Dua S, Bhuker M, Sharma P, Dhall M, Kapoor S (2014) Body mass index relates to blood pressure among adults. N Am J Med Sci 6: 89-95.

23. Drøyvold WB, Midthjell K, Nilsen TI, Holmen J (2005) Change in body mass index and its impact on blood pressure: a prospective population study. Int J Obes (Lond) 29: 650-655.

24. Elflein HM, Pfeiffer N, Hoffmann EM, Hoehn R, Kottler U, et al. (2014) Correlations between central corneal thickness and general anthropometric characteristics and cardiovascular parameters in a large European cohort from the Gutenberg Health Study. Cornea 33: 359-365. 